\title{
Another Class of Distances and Continuous Quasi-Distances in Product Spaces
}

\author{
Catherine Peppo \\ CFA-Université Pierre et Marie Curie, 4 place Jussieu, Casier 232, 75252 Paris Cedex 05, France \\ Correspondence should be addressed to Catherine Peppo; catherine.peppo@upmc.fr
}

Received 31 May 2013; Accepted 28 November 2013; Published 18 March 2014

Academic Editor: Dumitru Motreanu

Copyright (C) 2014 Catherine Peppo. This is an open access article distributed under the Creative Commons Attribution License, which permits unrestricted use, distribution, and reproduction in any medium, provided the original work is properly cited.

\begin{abstract}
We construct a class of continuous quasi-distances in a product of metric spaces and show that, generally, when the parameter $\lambda$ (as shown in the paper) is positive, $d$ is a distance and when $\lambda<0, d$ is only a continuous quasi-distance, but not a distance. It is remarkable that the same result in relation to the sign of $\lambda$ was found for two other classes of continuous quasi-distances (see Peppo (2010a, 2010b) and Peppo (2011)). This conclusion is due to the fact that $E$ is a product space. For the purposes of our main result, a notion of density in metric spaces is introduced.
\end{abstract}

\section{Introduction}

In this paper a quasi-distance $d$ on a set $E$ is defined as a function $d: E^{2} \mapsto[0 ;+\infty$ [ with the usual properties of a metric and a weaker version of the triangle inequality:

$$
d(x, y) \leq k[d(x, z)+d(y, z)]
$$

where $k \geq 1$.

This function is not always continuous with respect to the $d$-topology generated by itself in the same manner as by a distance. This is because the "open" balls $B(a, r)=\{x \in$ $E: d(x, a)<r\}$, which form a base for a complete system of neighbourhoods of $a \in E$, are not always open sets in the $d$ topology. Examples of "open" balls that are not open sets can be found in [1].

It is known that for every quasi-distance $d$, there exists another quasi-distance $d^{\prime}$, whose topology and uniformity are the same as those of $d$ and the open $d^{\prime}$-balls are open sets [2].

But if any condition or relation is satisfied with respect to a quasi-distance $d$, we do not know if, in general, the same condition or relation will be satisfied with respect to the "new" quasi-distance $d^{\prime}$, even if $d$ is equivalent to $d^{\prime}$.

For this reason, the continuity of a quasi-distance cannot be omitted without "danger."
In fixed-point theory, some authors require the continuity of the used quasi-distance as complementary condition (see, among others, [3]).

The most interesting fact about the quasi-metric spaces is that, in many applications, they constitute a more general setting than the metric spaces without losing the good properties of these last spaces (see, among others, [4]).

In [5-7] we proved that, generally, the functions defined in a product space for two points, $P\left(x_{1}, x_{2}, \ldots, x_{n}\right)$ and $Q\left(y_{1}, y_{2}, \ldots, y_{n}\right)$, respectively, by

$$
\begin{gathered}
d(P, Q)= \begin{cases}\sum_{i=1}^{n} a_{i} d_{i}\left(x_{i}, y_{i}\right) & \text { if } \sum_{i=1}^{n} \beta_{i} d_{i}\left(x_{i}, y_{i}\right) \geq 0, \\
\sum_{i=1}^{n} a_{i}^{\prime} d_{i}\left(x_{i}, y_{i}\right) & \text { if } \sum_{i=1}^{n} \beta_{i} d_{i}\left(x_{i}, y_{i}\right)<0,\end{cases} \\
d(P, Q)= \begin{cases}\frac{\sum_{i=1}^{n} a_{i} d_{i}\left(x_{i}, y_{i}\right)}{1+\sum_{i=1}^{n} a_{i} d_{i}\left(x_{i}, y_{i}\right)} & \text { if } \sum_{i=1}^{n} \beta_{i} d_{i}\left(x_{i}, y_{i}\right) \geq 0, \\
\frac{\sum_{i=1}^{n} a_{i}^{\prime} d_{i}\left(x_{i}, y_{i}\right)}{1+\sum_{i=1}^{n} a_{i}^{\prime} d_{i}\left(x_{i}, y_{i}\right)} & \text { if } \sum_{i=1}^{n} \beta_{i} d_{i}\left(x_{i}, y_{i}\right)<0,\end{cases}
\end{gathered}
$$

with $a_{i}-a_{i}^{\prime}=\lambda \beta_{i}$ for all $i \in I$, are distances when $\lambda>0$ but only continuous quasi-distances when $\lambda<0$. 
In this paper we revisit this problem for another family of functions in a product space defined by

$$
d(P, Q)= \begin{cases}\sqrt{\sum_{i=1}^{n} a_{i}^{2} d_{i}^{2}\left(x_{i}, y_{i}\right)} & \text { if } \sum_{i=1}^{n} \beta_{i} d_{i}^{2}\left(x_{i}, y_{i}\right) \geq 0, \\ \sqrt{\sum_{i=1}^{n} a_{i}^{\prime 2} d_{i}^{2}\left(x_{i}, y_{i}\right)} & \text { if } \sum_{i=1}^{n} \beta_{i} d_{i}^{2}\left(x_{i}, y_{i}\right)<0,\end{cases}
$$

with $a_{i}^{2}-a_{i}^{\prime 2}=\lambda \beta_{i}$ and we find the same result.

This makes us think that the conclusions reached are not coincidental.

We have reason to believe that a similar result holds for other classes of quasi-distances in product spaces.

Additionally, we notice that the iterated quasi-triangle constants of our quasi-distances,

$$
d\left(P_{1}, P_{n}\right) \leq k\left[d\left(P_{1}, P_{2}\right)+d\left(P_{2}, P_{3}\right)+\cdots+d\left(P_{n-1}, P_{n}\right)\right],
$$

which we are calling generalized improved quasi-triangle inequality, improve the general one:

$$
\begin{aligned}
d\left(P_{1}, P_{n}\right) \leq & k d\left(P_{1}, P_{2}\right)+k^{2} d\left(P_{2}, P_{3}\right) \\
& +\cdots+k^{n-3} d\left(P_{n-3}, P_{n-2}\right) \\
& +k^{n-2} d\left(P_{n-2}, P_{n-1}\right)+k^{n-2} d\left(P_{n-1}, P_{n}\right)
\end{aligned}
$$

(the two last coefficients are $k^{n-2}$; it is not a mistake).

In Section 2 we introduce the notion of a density condition in metric spaces for the purposes of our main result.

In Section 4 we give a counterexample proving the importance of the $\left(\alpha, \sqrt{-\beta_{i} / \beta_{j}}\right)$-density condition in part III of our main result.

\section{Density in Metric Spaces}

In the third part of our main result (Section 3) we will need metric spaces satisfying an additional property of density that we are calling $(\alpha, k)$-density condition.

Definition 1. For an $\alpha \in] 0 ; 1$ [ and a positive number $k$, the pair of metric spaces $(E, d)$ and $\left(E^{\prime}, d^{\prime}\right)$ satisfies the $(\alpha, k)$ density condition if the space $E$ contains at least three distinct points, $x, y, z$, satisfying the relations

$$
d(x, z)=\alpha d(x, y), \quad d(y, z)=(1-\alpha) d(x, y)
$$

and for every $\beta \in] \alpha ; 1$ [ there exist at least three distinct points $x^{\prime}, y^{\prime}, z^{\prime} \in E^{\prime}$, satisfying the relations

$$
\begin{gathered}
d^{\prime}\left(x^{\prime}, z^{\prime}\right)=\beta d^{\prime}\left(x^{\prime}, y^{\prime}\right), \\
d^{\prime}\left(y^{\prime}, z^{\prime}\right)=(1-\beta) d^{\prime}\left(x^{\prime}, y^{\prime}\right), \\
d^{\prime}\left(x^{\prime}, y^{\prime}\right)=k d(x, y) .
\end{gathered}
$$

In terms of segments, Definition 1 may be expressed as follows.

For an $\alpha \in] 0 ; 1[$ and a positive number $k$, the pair of metric spaces $(E, d)$ and $\left(E^{\prime}, d^{\prime}\right)$ satisfies the $(\alpha, k)$-density condition if $E$ contains at least a segment, $[x, y]$, containing a point $z$ dividing it at the ratio $\alpha: 1-\alpha$ and, for every $\beta \in] \alpha ; 1\left[, E^{\prime}\right.$ contains at least a segment $\left[x^{\prime}, y^{\prime}\right], k$ time "longer" in $\left(E^{\prime}, d^{\prime}\right)$ than $[x, y]$ in $(E, d)$ and containing a point $z^{\prime}$ dividing it at the ratio $\beta: 1-\beta$.

By segment we mean the set defined by the following:

Definition 2. For two points $x, y$ in a metric space $(E, d)$, the segment $[x ; y]$ is the set of points $z \in E$ satisfying

$$
d(x, y)=d(x, z)+d(z, y) .
$$

It is obvious that the extremities $x$ and $y$ belong to the segment $[x ; y]$, but it may only be reduced at its two extremities.

We note also that, despite appearances, if $u \in[x ; y]$, $v \in[x ; y]$, and $d(x, u)=d(x, v)$, this does not mean, in general, that $u=v$, as in the example below (of course, in the particular case when $d(x, u)=d(x, v)=0$, we have $u=v=x)$.

Example 3. Let $a, b$ be two strictly positive numbers satisfying $\max (a, b) \leq \min (2 a, 2 b), c=a+b, c_{1}$ a number satisfying $\max (a, b) \leq c_{1} \leq \min (2 a, 2 b)$, and $E$ a set containing whatever four points $A\left(x_{1}, y_{1}\right), B\left(x_{2}, y_{2}\right), C\left(x_{3}, y_{3}\right)$, and $D\left(x_{4}, y_{4}\right)$ of $R^{2}$. Define the function $d$ by

$$
\begin{aligned}
& d(A, B)=d(B, A)=c, \quad d(A, C)=d(C, A)=b, \\
& d(B, C)=d(C, B)=a, \\
& d(A, D)=d(D, A)=b, \quad d(B, D)=d(D, B)=a, \\
& d(C, D)=d(D, C)=c_{1}, \\
& \quad d(A, A)=d(B, B)=d(C, C)=d(D, D)=0 .
\end{aligned}
$$

The two first properties of a distance are obviously satisfied.

So, to prove that $d$ is a distance, it suffices to prove the triangle inequality in all possible "triangles" formed with the points of $E$, that is, $A B C, A B D, A C D$, and $B C D$ (Figure 1).

In the "triangles" $A B C$ and $A B D$, the "sides" are $a, b, c$ and the greatest one is $c=a+b$, so the "triangle" inequality is satisfied.

In the "triangles" $A C D$ and $B C D$, the "sides" are, respectively, $b, b, c_{1}$ and $a, a, c_{1}$ and the greatest one is, by our choice, $c_{1}$; or $c_{1} \leq \min (2 a, 2 b) \leq 2 a$, and $c_{1} \leq \min (2 a, 2 b) \leq$ $2 b$, so the "triangle" inequality is satisfied.

The points $C$ and $D$ belong to $[A, B]$, because $d(C, A)+$ $d(C, B)=b+a=c=d(A, B)$ and $d(D, A)+d(D, B)=b+a=$ $c=d(A, B)$. On the other hand $d(C, A)=d(D, A)=b$, but, generally, $C \neq D$.

We note that the $(\alpha, k)$-density condition, for an $\alpha \in] 0 ; 1[$ and a positive number $k$, as it is required in the third part of 


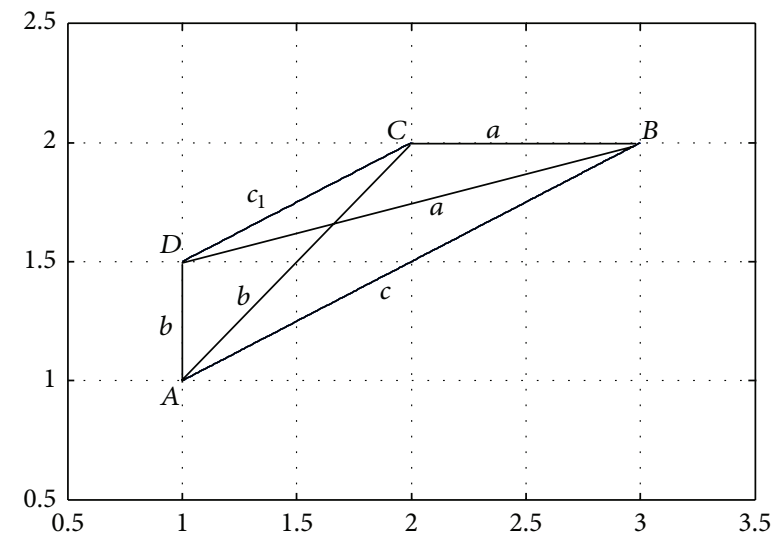

FIGURE 1



Figure 2

our main result (Section 3), is not a very restrictive condition. For example, if $d$ is the usual distance in $R^{2}, \alpha=1 / 3$, and $k=2$, in order that the pair $\left((E, d) ;\left(E^{\prime}, d^{\prime}\right)\right)$ satisfies the $(1 / 3,2)$-density condition, it suffices that $(E, d)$ contains the set $\{(1 ; 1),(2 ; 1),(4 ; 1)\}$, while $\left(E^{\prime}, d^{\prime}\right)$ contains the set $\{(1 ; 2)\} \bigcup\{(x ; 2): 3 \leq x \leq 7\}$ as in Figure 2 .

Clearly, every pair of normed vector spaces over $R$ satisfies the $(\alpha, k)$-density condition for each $\alpha \in] 0 ; 1[$ and for each positive number $k$.

\section{Main Result}

Theorem 4. Let $\left(E_{i}, d_{i}\right)$ for $i \in\{1,2, \ldots, n\}$ be metric spaces, $\lambda$ and $\beta_{i}$ real numbers, $a_{i}$ and $a_{i}^{\prime}$ positive numbers satisfying $a_{i}^{2}-a_{i}^{\prime 2}=\lambda \beta_{i}$, and $P\left(x_{1}, x_{2}, \ldots, x_{n}\right)$ and $Q\left(y_{1}, y_{2}, \ldots, y_{n}\right)$ two arbitrary points of $E=\prod_{i=1}^{n} E_{i}$. Then

(i) the functiond: $E^{2} \mapsto R^{+}$defined by

$$
d(P, Q)= \begin{cases}\sqrt{\sum_{i=1}^{n} a_{i}^{2} d_{i}^{2}\left(x_{i}, y_{i}\right)} & \text { if } \sum_{i=1}^{n} \beta_{i} d_{i}^{2}\left(x_{i}, y_{i}\right) \geq 0 \\ \sqrt{\sum_{i=1}^{n} a_{i}^{\prime 2} d_{i}^{2}\left(x_{i}, y_{i}\right)} & \text { if } \sum_{i=1}^{n} \beta_{i} d_{i}^{2}\left(x_{i}, y_{i}\right)<0\end{cases}
$$

is a continuous quasi-distance on $E$ satisfying the generalized improved triangle inequality for every $m$ points;

(ii) if $\geq 0$ or $\beta_{i}$ s are all nonnegative or all nonpositive, $d$ is a distance;

(iii) if $\lambda<0$ and there exist two indexes $(i, j) \in\{1,2, \ldots, n\}$ with $\beta_{i}<0, \beta_{j}>0$ and an $\left.\alpha \in\right] 0 ; 1[$ such that the spaces $E_{i}$ and $E_{j}$ satisfy the $\left(\alpha, \sqrt{-\beta_{i} / \beta_{j}}\right)$-density condition, the continuous quasi-distance $d$ is not a distance.

Proof. (i) First, we will prove that $d$ is a quasi-distance and then that $d$ is a continuous function of its two variables with respect to the $d$-topology.

It is clear that $d(P, Q) \geq 0$, that $d$ is symmetric, and that $d(P, Q)=0 \Leftrightarrow P=Q$.

Let $K_{1}=\min \left\{a_{i}, a_{i}^{\prime}\right\}_{1 \leq i \leq n}, K_{2}=\max \left\{a_{i}, a_{i}^{\prime}\right\}_{1 \leq i \leq n}$, and $D(P, Q)=\sqrt{\sum_{i=1}^{n} d_{i}^{2}\left(x_{i}, y_{i}\right)}$.

Clearly, for whatever points $P$ and $Q$, we have

$$
K_{1} D(P, Q) \leq d(P, Q) \leq K_{2} D(P, Q) .
$$

As $D$ is a distance, we can write, for whatever points $\quad P_{1}\left(x_{1}^{(1)}, x_{2}^{(1)}, \ldots, x_{n}^{(1)}\right), \quad P_{2}\left(x_{1}^{(2)}, x_{2}^{(2)}, \ldots, x_{n}^{(2)}\right), \ldots$, $P_{i}\left(x_{1}^{(i)}, x_{2}^{(i)}, \ldots, x_{n}^{(i)}\right)$,

$$
\begin{aligned}
d\left(P_{1}, P_{i}\right) & \leq K_{2} D\left(P_{1}, P_{i}\right) \\
& \leq K_{2}\left[D\left(P_{1}, P_{2}\right)+D\left(P_{2}, P_{3}\right)+\cdots+D\left(P_{i-1}, P_{i}\right)\right] \\
& \leq \frac{K_{2}}{K_{1}}\left[d\left(P_{1}, P_{2}\right)+d\left(P_{2}, P_{3}\right)+\cdots+d\left(P_{i-1}, P_{i}\right)\right] .
\end{aligned}
$$

This means that $d$ is a quasi-distance with constant $K=$ $K_{2} / K_{1}$, and the same constant holds for whatever $m$ points. Let us show now that $d$ is continuous with respect to the $d$ topology.

Inequalities (11) show that $d$ and $D$ are topologically equivalent. Hence to prove that $d$ is continuous, we will show that for every two sequences, $P_{k}\left(x_{1}^{(k)}, x_{2}^{(k)}, \ldots, x_{n}^{(k)}\right)$ and $Q_{k}\left(y_{1}^{(k)}, y_{2}^{(k)}, \ldots, y_{n}^{(k)}\right)$, converging, respectively, to $P\left(x_{1}, x_{2}, \ldots, x_{n}\right)$ and $Q\left(y_{1}, y_{2}, \ldots, y_{n}\right)$ for $k \rightarrow+\infty$, $d\left(P_{k}, Q_{k}\right)$ converges to $d(P, Q)$.

For the points $P\left(x_{1}, x_{2}, \ldots, x_{n}\right)$ and $Q\left(y_{1}, y_{2}, \ldots, y_{n}\right)$, in the following, we will denote $g(P, Q)=\sqrt{\sum_{i=1}^{n} a_{i}^{2} d_{i}^{2}\left(x_{i}, y_{i}\right)}$, $h(P, Q)=\sqrt{\sum_{i=1}^{n} a_{i}^{\prime 2} d_{i}^{2}\left(x_{i}, y_{i}\right)}$, and $A=\sum_{i=1}^{n} \beta_{i} d_{i}^{2}\left(x_{i}, y_{i}\right)$.

If $A>0(A<0)$, an integer $p$ exists such that for $k \in N$, $k \geq p$ we have $\sum_{i=1}^{n} \beta_{i} d_{i}^{2}\left(x_{i}^{(k)}, y_{i}^{(k)}\right)>0\left(\sum_{i=1}^{n} \beta_{i} d_{i}^{2}\left(x_{i}^{(k)}, y_{i}^{(k)}\right)<\right.$ $0)$ (because the sequence $\sum_{i=1}^{n} \beta_{i} d_{i}^{2}\left(x_{i}^{(k)}, y_{i}^{(k)}\right)$ converges to $\left.A\right)$.

For $k \in N, k \geq p$, we have $d\left(P_{k}, Q_{k}\right)=g\left(P_{k}, Q_{k}\right)$ $\left(d\left(P_{k}, Q_{k}\right)=h\left(P_{k}, Q_{k}\right)\right)$ and, consequently, $d\left(P_{k}, Q_{k}\right)$ converges to $g(P, Q)=d(P, Q)$ and $(h(P, Q)=d(P, Q))$. 
If $A=0$, from $a_{i}^{2}-a_{i}^{\prime 2}=\lambda \beta_{i}$ we deduce

$$
\begin{aligned}
\sqrt{\sum_{i=1}^{n} a_{i}^{2} d_{i}^{2}\left(x_{i}, y_{i}\right)} & =\sqrt{\sum_{i=1}^{n}\left(a_{i}^{\prime 2}+\lambda \beta_{i}\right) d_{i}^{2}\left(x_{i}, y_{i}\right)} \\
& =\sqrt{\sum_{i=1}^{n} a_{i}^{\prime 2} d_{i}^{2}\left(x_{i}, y_{i}\right)+\lambda A} \\
& =\sqrt{\sum_{i=1}^{n} a_{i}^{\prime 2} d_{i}^{2}\left(x_{i}, y_{i}\right) .}
\end{aligned}
$$

That is, $g(P, Q)=h(P, Q)$.

It follows that $g\left(P_{k}, Q_{k}\right)$ and $h\left(P_{k}, Q_{k}\right)$ converge to the same limit $g(P, Q)=h(P, Q)=d(P, Q)$. We can conclude that $d\left(P_{k}, Q_{k}\right)$, formed by the terms of the two sequences above, also converges to $d(P, Q)$. Thus, $d$ is continuous and the "open" balls $B\left(P_{0}, r\right)=\left\{P \in E: d\left(P, P_{0}\right)<r\right\}$ really are, in fact, open sets for the $d$-topology.

(ii) If $\beta_{i}$ s are all nonnegative, $d(P, Q)=g(P, Q)$ for every $P$ and $Q$; if $\beta_{i}$ s are all nonpositive, $d(P, Q)=h(P, Q)$ for every $P$ and $Q$ (recall that if $\sum_{i=1}^{n} \beta_{i} d_{i}^{2}\left(x_{i}, y_{i}\right)=0, d(P, Q)=$ $g(P, Q)=h(P, Q))$. As $g$ and $h$ are distances, $d$ is a distance too.

If $\lambda=0, a_{i}=a_{i}^{\prime}$ for every $i \in\{1,2, \ldots, n\}$, so $d(P, Q)=$ $g(P, Q)=h(P, Q)$ for every $P$ and $Q$; that is, $d$ is a distance.

Suppose now that $\lambda>0$; for two arbitrary points $P\left(x_{1}, x_{2}, \ldots, x_{n}\right)$ and $Q\left(y_{1}, y_{2}, \ldots, y_{n}\right)$, denoting again $A=$ $\sum_{i=1}^{n} \beta_{i} d_{i}^{2}\left(x_{i}, y_{i}\right)$ we have

$$
\begin{aligned}
g(P, Q) & =\sqrt{\sum_{i=1}^{n} a_{i}^{2} d_{i}^{2}\left(x_{i}, y_{i}\right)}=\sqrt{\sum_{i=1}^{n}\left(a_{i}^{\prime 2}+\lambda \beta_{i}\right) d_{i}^{2}\left(x_{i}, y_{i}\right)} \\
& =\sqrt{\sum_{i=1}^{n} a_{i}^{\prime 2} d_{i}^{2}\left(x_{i}, y_{i}\right)+\lambda A .}
\end{aligned}
$$

So, for $\lambda>0$ it follows that

$$
\begin{aligned}
& A \geq 0 \Longrightarrow g(P, Q) \geq h(P, Q), \\
& A<0 \Longrightarrow g(P, Q)<h(P, Q) .
\end{aligned}
$$

This means that if $\lambda>0, d=\max (g, h)$, and since the maximum of two distances is a distance, $d$ is a distance.

Notice that in this part of the theorem we did not need any supplementary density condition on the spaces $\left(E_{i}, d_{i}\right)$.

(iii) Suppose now that $\lambda<0$ and that there exist an $\alpha \in$ ]0; 1 [ and two indexes $i, j \in\{1,2, \ldots, n\}$ such that $\beta_{i}<0$, $\beta_{j}>0$, and the pair $\left(E_{i}, E_{j}\right)$ satisfies the $\left(\alpha, \sqrt{-\beta_{i} / \beta_{j}}\right)$-density condition.
We choose a $\beta \in] \alpha ; 1[$ satisfying the relation

$$
\begin{aligned}
-\beta_{i} \beta_{j} a_{j}^{2} a_{i}^{2}(\beta-\alpha) & \\
< & \lambda^{2} \beta_{i}^{2} \beta_{j}^{2}(\beta-\alpha)\left(1-\frac{\alpha+\beta}{2}\right)^{2} \\
& +2\left(-\beta_{i} a_{j}^{2} \beta+\beta_{j} a_{i}^{2} \alpha\right) \lambda \beta_{i} \beta_{j}\left(1-\frac{\alpha+\beta}{2}\right) .
\end{aligned}
$$

Such a $\beta>\alpha$ exists because, passing into limit in (16) as $\beta \rightarrow$ $\alpha^{+}$, we obtain

$$
0<2 \lambda \beta_{i} \beta_{j}(1-\alpha) \alpha\left(\beta_{j} a_{i}^{2}-\beta_{i} a_{j}^{2}\right)
$$

that is clearly true.

As $\left(E_{i}, E_{j}\right)$ satisfies the $\left(\alpha, \sqrt{-\beta_{i} / \beta_{j}}\right)$-density condition, there exist three points $x_{i}, y_{i}, z_{i} \in E_{i}$, satisfying the relations $d_{i}\left(x_{i}, z_{i}\right)=\alpha d_{i}\left(x_{i}, y_{i}\right)$ and $d_{i}\left(y_{i}, z_{i}\right)=(1-\alpha) d_{i}\left(x_{i}, y_{i}\right)$, and three points $x_{j}, y_{j}, z_{j} \in E_{j}$, satisfying the relations

$$
\begin{gathered}
d_{j}\left(x_{j}, z_{j}\right)=\beta d_{j}\left(x_{j}, y_{j}\right), \\
d_{j}\left(y_{j}, z_{j}\right)=(1-\beta) d_{j}\left(x_{j}, y_{j}\right), \\
d_{j}\left(x_{j}, y_{j}\right)=\sqrt{-\frac{\beta_{i}}{\beta_{j}}} d_{i}\left(x_{i}, y_{i}\right) .
\end{gathered}
$$

With the points $x_{i}, y_{i}, z_{i} \in E_{i}$ and $x_{j}, y_{j}, z_{j} \in E_{j}$ we construct the points $P\left(\ldots, x_{i}, \ldots, x_{j}, \ldots\right), Q\left(\ldots, y_{i}, \ldots, y_{j}, \ldots\right)$, and $R\left(\ldots, z_{i}, \ldots, z_{j}, \ldots\right)$ of $E$ (except the $i$ th and the $j$ th coordinates, all the other coordinates are equal in $P, Q$, and $R$ ) and will prove that

$$
d(P, Q)>d(P, R)+d(Q, R) .
$$

For the points $P$ and $Q$ we have from (18) that

$$
\sum_{k=1}^{n} \beta_{k} d_{k}^{2}\left(x_{k}, y_{k}\right)=\beta_{i} d_{i}^{2}\left(x_{i}, y_{i}\right)+\beta_{j} d_{j}^{2}\left(x_{j}, y_{j}\right)=0
$$

$$
\begin{aligned}
& \text { so } d(P, Q)=\sqrt{a_{i}^{2} d_{i}^{2}\left(x_{i}, y_{i}\right)+a_{j}^{2} d_{j}^{2}\left(x_{j}, y_{j}\right)}= \\
& \sqrt{\left(a_{i}^{2}-\left(\beta_{i} / \beta_{j}\right) a_{j}^{2}\right)} d_{i}\left(x_{i}, y_{i}\right) \text {. } \\
& \text { For the points } P \text { and } R \text {, } \\
& \begin{aligned}
\sum_{k=1}^{n} \beta_{k} d_{k}^{2}\left(x_{k}, z_{k}\right) & =\beta_{i} d_{i}^{2}\left(x_{i}, z_{i}\right)+\beta_{j} d_{j}^{2}\left(x_{j}, z_{j}\right) \\
& =\beta_{i} \alpha^{2} d_{i}^{2}\left(x_{i}, y_{i}\right)+\beta_{j} \beta^{2}\left(-\frac{\beta_{i}}{\beta_{j}}\right) d_{i}^{2}\left(x_{i}, y_{i}\right) \\
& =\beta_{i} d_{i}^{2}\left(x_{i}, y_{i}\right)\left(\alpha^{2}-\beta^{2}\right)>0,
\end{aligned}
\end{aligned}
$$

because $\beta_{i}<0$ and $0<\alpha<\beta$, so $d(P, R)=$ $\sqrt{a_{i}^{2} d_{i}^{2}\left(x_{i}, z_{i}\right)+a_{j}^{2} d_{j}^{2}\left(x_{j}, z_{j}\right)}=\sqrt{\left(a_{i}^{2} \alpha^{2}-a_{j}^{2} \beta^{2}\left(\beta_{i} / \beta_{j}\right)\right)} d_{i}\left(x_{i}\right.$, $\left.y_{i}\right)$. 
For the points $Q$ and $R$,

$$
\begin{aligned}
\sum_{k=1}^{n} \beta_{k} d_{k}^{2}\left(x_{k}, y_{k}\right)= & \beta_{i} d_{i}^{2}\left(y_{i}, z_{i}\right)+\beta_{j} d_{j}^{2}\left(y_{j}, z_{j}\right) \\
= & \beta_{i}(1-\alpha)^{2} d_{i}^{2}\left(x_{i}, y_{i}\right) \\
& -\beta_{j}(1-\beta)^{2} \frac{\beta_{i}}{\beta_{j}} d_{i}^{2}\left(x_{i}, y_{i}\right) \\
= & \beta_{i} d_{i}^{2}\left(x_{i}, y_{i}\right)\left[(1-\alpha)^{2}-(1-\beta)^{2}\right]<0,
\end{aligned}
$$

because $\beta_{i}<0$ and $(1-\alpha)^{2}>(1-\beta)^{2}$, so $d(Q, R)=\sqrt{a_{i}^{\prime 2} d_{i}^{2}\left(y_{i}, z_{i}\right)+a_{j}^{\prime 2} d_{j}^{2}\left(y_{j}, z_{j}\right)}=$ $\sqrt{a_{i}^{\prime 2}(1-\alpha)^{2}-a_{j}^{\prime 2}(1-\beta)^{2}\left(\beta_{i} / \beta_{j}\right)} d_{i}\left(x_{i}, y_{i}\right)$.

We are ready to prove now the inequality $d(P, Q)>$ $d(P, R)+d(Q, R)$, transforming it equivalently to

$$
\begin{aligned}
\sqrt{a_{i}^{2}-\frac{\beta_{i}}{\beta_{j}} a_{j}^{2}} d_{i}\left(x_{i}, y_{i}\right)> & \sqrt{a_{i}^{2} \alpha^{2}-a_{j}^{2} \beta^{2} \frac{\beta_{i}}{\beta_{j}}} d_{i}\left(x_{i}, y_{i}\right) \\
& +\sqrt{a_{i}^{\prime 2}(1-\alpha)^{2}-a_{j}^{\prime 2}(1-\beta)^{2} \frac{\beta_{i}}{\beta_{j}}} \\
& \times d_{i}\left(x_{i}, y_{i}\right) \\
\Longleftrightarrow \sqrt{a_{i}^{2}-\frac{\beta_{i}}{\beta_{j}} a_{j}^{2}}> & \sqrt{a_{i}^{2} \alpha^{2}-a_{j}^{2} \beta^{2} \frac{\beta_{i}}{\beta_{j}}} \\
& +\sqrt{a_{i}^{\prime 2}(1-\alpha)^{2}-a_{j}^{\prime 2}(1-\beta)^{2} \frac{\beta_{i}}{\beta_{j}}} \\
\Longleftrightarrow \sqrt{\beta_{j} a_{i}^{2}-\beta_{i} a_{j}^{2}}> & \sqrt{\beta_{j} a_{i}^{2} \alpha^{2}-\beta_{i} a_{j}^{2} \beta^{2}} \\
& +\sqrt{\beta_{j} a_{i}^{\prime 2}(1-\alpha)^{2}-\beta_{i} a_{j}^{\prime 2}(1-\beta)^{2}} .
\end{aligned}
$$

From $\beta_{j} a_{i}^{2}>\beta_{j} a_{i}^{2} \alpha^{2}$ and $-\beta_{i} a_{j}^{2}>-\beta_{i} a_{j}^{2} \beta^{2}$, we have $\sqrt{\beta_{j} a_{i}^{2}-\beta_{i} a_{j}^{2}}>\sqrt{\beta_{j} a_{i}^{2} \alpha^{2}-\beta_{i} a_{j}^{2} \beta^{2}}$, so $\sqrt{\beta_{j} a_{i}^{2}-\beta_{i} a_{j}^{2}}-$ $\sqrt{\beta_{j} a_{i}^{2} \alpha^{2}-\beta_{i} a_{j}^{2} \beta^{2}}$ is positive and we can equivalently square

$$
\begin{gathered}
\Longleftrightarrow\left(\sqrt{\beta_{j} a_{i}^{2}-\beta_{i} a_{j}^{2}}-\sqrt{\beta_{j} a_{i}^{2} \alpha^{2}-\beta_{i} a_{j}^{2} \beta^{2}}\right)^{2} \\
>\left(\sqrt{\beta_{j} a_{i}^{\prime 2}(1-\alpha)^{2}-\beta_{i} a_{j}^{\prime 2}(1-\beta)^{2}}\right)^{2} \\
\Longleftrightarrow \beta_{j} a_{i}^{2}-\beta_{i} a_{j}^{2}+\beta_{j} a_{i}^{2} \alpha^{2}-\beta_{i} a_{j}^{2} \beta^{2} \\
-2 \sqrt{\beta_{j} a_{i}^{2}-\beta_{i} a_{j}^{2}} \sqrt{\beta_{j} a_{i}^{2} \alpha^{2}-\beta_{i} a_{j}^{2} \beta^{2}} \\
>\beta_{j} a_{i}^{\prime 2}(1-\alpha)^{2}-\beta_{i} a_{j}^{\prime 2}(1-\beta)^{2}
\end{gathered}
$$

(because $a_{i}^{\prime 2}=a_{i}^{2}-\lambda \beta_{i}$ and $a_{j}^{\prime 2}=a_{j}^{2}-\lambda \beta_{j}$ ),

$$
\begin{aligned}
& \Longleftrightarrow-2 \sqrt{\beta_{j} a_{i}^{2}-\beta_{i} a_{j}^{2}} \sqrt{\beta_{j} a_{i}^{2} \alpha^{2}-\beta_{i} a_{j}^{2} \beta^{2}} \\
&> \beta_{j} a_{i}^{2}(1-\alpha)^{2}+\lambda \beta_{i} \beta_{j}(1-\beta)^{2}-\beta_{j} a_{i}^{2} \\
&-\beta_{j} a_{i}^{2} \alpha^{2}-\lambda \beta_{i} \beta_{j}(1-\alpha)^{2}-\beta_{i} a_{j}^{2}(1-\beta)^{2} \\
&+\beta_{i} a_{j}^{2}+\beta_{i} a_{j}^{2} \beta^{2} \\
& \Longleftrightarrow- \sqrt{\beta_{j} a_{i}^{2}-\beta_{i} a_{j}^{2}} \sqrt{\beta_{j} a_{i}^{2} \alpha^{2}-\beta_{i} a_{j}^{2} \beta^{2}} \\
&> \beta_{j} a_{i}^{2}\left[(1-\alpha)^{2}-1-\alpha^{2}\right] \\
&+\lambda \beta_{i} \beta_{j}\left[(1-\beta)^{2}-(1-\alpha)^{2}\right] \\
&-\beta_{i} a_{j}^{2}\left[(1-\beta)^{2}-1-\beta^{2}\right] \\
& \Longleftrightarrow- \sqrt{\beta_{j} a_{i}^{2}-\beta_{i} a_{j}^{2}} \sqrt{\beta_{j} a_{i}^{2} \alpha^{2}-\beta_{i} a_{j}^{2} \beta^{2}} \\
&> \beta_{i} a_{j}^{2} 2 \beta-\beta_{j} a_{i}^{2} 2 \alpha+\lambda \beta_{i} \beta_{j}(\alpha-\beta)(2-\alpha-\beta) \\
&< \sqrt{\beta_{j} a_{i}^{2}-\beta_{i} a_{j}^{2}} \sqrt{\beta_{j} a_{i}^{2} \alpha^{2}-\beta_{i} a_{j}^{2} \beta^{2}} \\
& \Longleftrightarrow a_{j}^{2} \beta+\beta_{j} a_{i}^{2} \alpha+\lambda \beta_{i} \beta_{j}(\beta-\alpha)\left(1-\frac{\alpha+\beta}{2}\right)
\end{aligned}
$$

(we square)

$$
\begin{aligned}
\Longleftrightarrow & \left(\beta_{j} a_{i}^{2}-\beta_{i} a_{j}^{2}\right)\left(\beta_{j} a_{i}^{2} \alpha^{2}-\beta_{i} a_{j}^{2} \beta^{2}\right) \\
< & \left(-\beta_{i} a_{j}^{2} \beta+\beta_{j} a_{i}^{2} \alpha\right)^{2} \\
& +\left[\lambda \beta_{i} \beta_{j}(\beta-\alpha)\left(1-\frac{\alpha+\beta}{2}\right)\right]^{2} \\
& +2\left(-\beta_{i} a_{j}^{2} \beta+\beta_{j} a_{i}^{2} \alpha\right) \lambda \beta_{i} \beta_{j}(\beta-\alpha)\left(1-\frac{\alpha+\beta}{2}\right) \\
\Longleftrightarrow \beta_{j}^{2} a_{i}^{4} \alpha^{2}-\beta_{i} \beta_{j} a_{j}^{2} a_{i}^{2} \alpha^{2}-\beta_{i} \beta_{j} a_{j}^{2} a_{i}^{2} \beta^{2}+\beta_{i}^{2} a_{j}^{4} \beta^{2} & \beta_{i}^{2} a_{j}^{4} \beta^{2}+\beta_{j}^{2} a_{i}^{4} \alpha^{2}-2 \beta_{i} \beta_{j} a_{i}^{2} a_{j}^{2} \alpha \beta \\
& +\lambda^{2} \beta_{i}^{2} \beta_{j}^{2}(\beta-\alpha)^{2}\left(1-\frac{\alpha+\beta}{2}\right)^{2} \\
& +2\left(-\beta_{i} a_{j}^{2} \beta+\beta_{j} a_{i}^{2} \alpha\right) \lambda \beta_{i} \beta_{j}(\beta-\alpha)\left(1-\frac{\alpha+\beta}{2}\right) \\
& +2\left(-\beta_{i} a_{j}^{2} \beta+\beta_{j} a_{i}^{2} \alpha\right) \lambda \beta_{i} \beta_{j}(\beta-\alpha)\left(1-\frac{\alpha+\beta}{2}\right) \\
< & -2 \beta_{i} \beta_{j} a_{j}^{2} a_{i}^{2} \alpha^{2} a_{i}^{2} a_{j}^{2} \alpha \beta+\beta_{i} \beta_{j} a_{j}^{2} a_{i}^{2} \beta^{2} \\
& \lambda^{2} \beta_{i}^{2} \beta_{j}^{2}(\beta-\alpha)^{2}\left(1-\frac{\alpha+\beta}{2}\right)^{2} \\
&
\end{aligned}
$$




$$
\begin{aligned}
\Longleftrightarrow & \beta_{i} \beta_{j} a_{j}^{2} a_{i}^{2}\left(2 \alpha \beta-\alpha^{2}-\beta^{2}\right) \\
< & \lambda^{2} \beta_{i}^{2} \beta_{j}^{2}(\beta-\alpha)^{2}\left(1-\frac{\alpha+\beta}{2}\right)^{2} \\
& +2\left(-\beta_{i} a_{j}^{2} \beta+\beta_{j} a_{i}^{2} \alpha\right) \lambda \beta_{i} \beta_{j} \\
& \times(\beta-\alpha)\left(1-\frac{\alpha+\beta}{2}\right) \\
\Longleftrightarrow & -\beta_{i} \beta_{j} a_{j}^{2} a_{i}^{2}(\beta-\alpha)^{2} \\
< & \lambda^{2} \beta_{i}^{2} \beta_{j}^{2}(\beta-\alpha)^{2}\left(1-\frac{\alpha+\beta}{2}\right)^{2} \\
& +2\left(-\beta_{i} a_{j}^{2} \beta+\beta_{j} a_{i}^{2} \alpha\right) \lambda \beta_{i} \beta_{j} \\
& \times(\beta-\alpha)\left(1-\frac{\alpha+\beta}{2}\right) .
\end{aligned}
$$

Dividing by $(\beta-\alpha)$ we have

$$
\begin{aligned}
\Longleftrightarrow & -\beta_{i} \beta_{j} a_{j}^{2} a_{i}^{2}(\beta-\alpha) \\
< & \lambda^{2} \beta_{i}^{2} \beta_{j}^{2}(\beta-\alpha)\left(1-\frac{\alpha+\beta}{2}\right)^{2} \\
& +2\left(-\beta_{i} a_{j}^{2} \beta+\beta_{j} a_{i}^{2} \alpha\right) \lambda \beta_{i} \beta_{j}\left(1-\frac{\alpha+\beta}{2}\right) .
\end{aligned}
$$

The last inequality is true from (16). The proof is over. So, $d$ is not a distance.

Example 5. For $P\left(x_{1}, x_{2}\right)$ and $Q\left(y_{1}, y_{2}\right) \in R^{2}$, the function defined by

$$
\begin{aligned}
& d(P, Q) \\
& = \begin{cases}\sqrt{2\left(x_{1}-y_{1}\right)^{2}+\left(x_{2}-y_{2}\right)^{2}} & \text { if } 2\left(x_{2}-y_{2}\right)^{2} \geq\left(x_{1}-y_{1}\right)^{2}, \\
\sqrt{\left(x_{1}-y_{1}\right)^{2}+3\left(x_{2}-y_{2}\right)^{2}} & \text { if } 2\left(x_{2}-y_{2}\right)^{2}<\left(x_{1}-y_{1}\right)^{2},\end{cases}
\end{aligned}
$$

is a continuous quasi-distance, but not a distance. Here, $\lambda=$ $-1, \beta_{1}=-1, \beta_{2}=2, a_{1}^{2}=2, a_{1}^{\prime 2}=1, a_{2}^{2}=1$, and $a_{2}^{\prime 2}=3$, while the function defined by

$$
\begin{aligned}
& d(P, Q) \\
& = \begin{cases}\sqrt{\left(x_{1}-y_{1}\right)^{2}+3\left(x_{2}-y_{2}\right)^{2}} & \text { if } 2\left(x_{2}-y_{2}\right)^{2} \geq\left(x_{1}-y_{1}\right)^{2}, \\
\sqrt{2\left(x_{1}-y_{1}\right)^{2}+\left(x_{2}-y_{2}\right)^{2}} & \text { if } 2\left(x_{2}-y_{2}\right)^{2}<\left(x_{1}-y_{1}\right)^{2},\end{cases}
\end{aligned}
$$

is a distance. $a_{2}^{\prime 2}=1$.

\section{A Counterexample}

Showing the importance of the $\left(\alpha, \sqrt{-\beta_{i} / \beta_{j}}\right)$-density condition in part III of the theorem.

Let $E_{1}=\left\{x_{1}, y_{1}\right\}, E_{2}=\left\{x_{2}, y_{2}\right\}$ be two metric spaces with distances, respectively, $d_{1}, d_{2}, E=E_{1} \times$ $E_{2}=\left\{P\left(x_{1}, x_{2}\right), Q\left(x_{1}, y_{2}\right), R\left(y_{1}, x_{2}\right), T\left(y_{1}, y_{2}\right)\right\}, x_{i} \neq y_{i}, \beta_{i}$, real numbers, and $a_{i}, a_{i}^{\prime}$ positive numbers satisfying, for $i=$ $1,2, a_{i}^{2}-a_{i}^{\prime 2}=\lambda \beta_{i}, \lambda<0, \beta_{1}<0, \beta_{2}>0$, and $\left(a_{1}^{2}-a_{1}^{\prime 2}\right)\left(a_{2}^{2}-\right.$ $\left.a_{2}^{\prime 2}\right)+4 a_{1}^{\prime 2} a_{2}^{2} \geq 0 \Leftrightarrow \lambda^{2} \beta_{1} \beta_{2}+4 a_{1}^{\prime 2} a_{2}^{2} \geq 0$.

For whatever $\alpha \in] 0 ; 1[$, it is obvious that the pair $\left(\left(E_{1}, d_{1}\right),\left(E_{2}, d_{2}\right)\right)$ does not satisfy the $\left(\alpha, \sqrt{-\beta_{i} / \beta_{j}}\right)$-density condition.

We will show that the function $d: E \times E \mapsto R^{+}$defined for every two points $M\left(z_{1}, z_{2}\right), N\left(t_{1}, t_{2}\right) \in E$ by

$$
d(M, N)=\left\{\begin{array}{l}
\sqrt{a_{1}^{2} d_{1}^{2}\left(z_{1}, z_{2}\right)+a_{2}^{2} d_{2}^{2}\left(t_{1}, t_{2}\right)} \\
\text { if } \beta_{1} d_{1}^{2}\left(z_{1}, z_{2}\right)+\beta_{2} d_{2}^{2}\left(t_{1}, t_{2}\right) \geq 0 \\
\sqrt{a_{1}^{\prime 2} d_{1}^{2}\left(z_{1}, z_{2}\right)+a_{2}^{\prime 2} d_{2}^{2}\left(t_{1}, t_{2}\right)} \\
\text { if } \beta_{1} d_{1}^{2}\left(z_{1}, z_{2}\right)+\beta_{2} d_{2}^{2}\left(t_{1}, t_{2}\right)<0
\end{array}\right.
$$

is a distance.

For every three points of $E$, we will show that the greatest "distance" (we put quotes because we do not yet know if $d$ is really a distance) between these points is inferior or equal to the sum of the two others.

4.1. First Case $\left(\beta_{1} d_{1}^{2}\left(x_{1}, y_{1}\right)+\beta_{2} d_{2}^{2}\left(x_{2}, y_{2}\right) \geq 0\right)$. The six "distances" are

$$
\begin{gathered}
d(P, Q)=d(R, T)=a_{2} d_{2}\left(x_{2}, y_{2}\right), \\
d(P, R)=d(Q, T)=a_{1}^{\prime} d_{1}\left(x_{1}, y_{1}\right), \\
d(Q, R)=d(P, T)=\sqrt{a_{1}^{2} d_{1}^{2}\left(x_{1}, y_{1}\right)+a_{2}^{2} d_{2}^{2}\left(x_{2}, y_{2}\right) .}
\end{gathered}
$$

As $a_{1}^{2}-a_{1}^{\prime 2}=\lambda \beta_{1}>0$, we have $a_{1}>a_{1}^{\prime}$, so

$\sqrt{a_{1}^{2} d_{1}^{2}\left(x_{1}, y_{1}\right)+a_{2}^{2} d_{2}^{2}\left(x_{2}, y_{2}\right)} \geq a_{1} d_{1}\left(x_{1}, y_{1}\right) \geq a_{1}^{\prime} d_{1}\left(x_{1}, y_{1}\right)$,

$$
\sqrt{a_{1}^{2} d_{1}^{2}\left(x_{1}, y_{1}\right)+a_{2}^{2} d_{2}^{2}\left(x_{2}, y_{2}\right)} \geq a_{2} d_{2}\left(x_{2}, y_{2}\right) \text {. }
$$

The greatest of the six "distances" is $d(Q, R)=d(P, T)=$ $\sqrt{a_{1}^{2} d_{1}^{2}\left(x_{1}, y_{1}\right)+a_{2}^{2} d_{2}^{2}\left(x_{2}, y_{2}\right)}$.

In each of the four possible "triangles," $P Q R$, $P Q T, P R T$, and $Q R T$, there is a side equal to $\sqrt{a_{1}^{2} d_{1}^{2}\left(x_{1}, y_{1}\right)+a_{2}^{2} d_{2}^{2}\left(x_{2}, y_{2}\right)}$, another is equal to $a_{1}^{\prime} d_{1}\left(x_{1}, y_{1}\right)$, and the third is $a_{2} d_{2}\left(x_{2}, y_{2}\right)$. 
Therefore we have to prove only one "triangle inequality," valid for the four triangles:

$$
\sqrt{a_{1}^{2} d_{1}^{2}\left(x_{1}, y_{1}\right)+a_{2}^{2} d_{2}^{2}\left(x_{2}, y_{2}\right)} \leq a_{1}^{\prime} d_{1}\left(x_{1}, y_{1}\right)+a_{2} d_{2}\left(x_{2}, y_{2}\right),
$$

or equivalently

$$
\begin{aligned}
a_{1}^{2} & d_{1}^{2}\left(x_{1}, y_{1}\right)+a_{2}^{2} d_{2}^{2}\left(x_{2}, y_{2}\right) \\
\leq & a_{1}^{\prime 2} d_{1}^{2}\left(x_{1}, y_{1}\right)+a_{2}^{2} d_{2}^{2}\left(x_{2}, y_{2}\right) \\
& +2 a_{1}^{\prime} a_{2} d_{1}\left(x_{1}, y_{1}\right) d_{2}\left(x_{2}, y_{2}\right) \\
& \Longleftrightarrow\left(a_{1}^{2}-a_{1}^{\prime 2}\right) d_{1}\left(x_{1}, y_{1}\right) \\
\leq & 2 a_{1}^{\prime} a_{2} d_{2}\left(x_{2}, y_{2}\right) \Longleftrightarrow \frac{d_{1}^{2}\left(x_{1}, y_{1}\right)}{d_{2}^{2}\left(x_{2}, y_{2}\right)} \\
\leq & \frac{4 a_{1}^{\prime 2} a_{2}^{2}}{\lambda^{2} \beta_{1}^{2}} .
\end{aligned}
$$

But from $\beta_{1} d_{1}^{2}\left(x_{1}, y_{1}\right)+\beta_{2} d_{2}^{2}\left(x_{2}, y_{2}\right) \geq 0$ we have $d_{1}^{2}\left(x_{1}, y_{1}\right) / d_{2}^{2}\left(x_{2}, y_{2}\right) \leq \beta_{2} /-\beta_{1}$ and from $\lambda^{2} \beta_{1} \beta_{2}+4 a_{1}^{\prime 2} a_{2}^{2} \geq 0$ we have $\beta_{2} /-\beta_{1} \leq 4 a_{1}^{\prime 2} a_{2}^{2} / \lambda^{2} \beta_{1}^{2}$, so $d_{1}^{2}\left(x_{1}, y_{1}\right) / d_{2}^{2}\left(x_{2}, y_{2}\right) \leq$ $4 a_{1}^{\prime 2} a_{2}^{2} / \lambda^{2} \beta_{1}^{2}$ and the triangle inequality is satisfied.

4.2. Second Case $\left(\beta_{1} d_{1}^{2}\left(x_{1}, y_{1}\right)+\beta_{2} d_{2}^{2}\left(x_{2}, y_{2}\right)<0\right)$. The six "distances" are

$$
\begin{gathered}
d(P, Q)=d(R, T)=a_{2} d_{2}\left(x_{2}, y_{2}\right), \\
d(P, R)=d(Q, T)=a_{1}^{\prime} d_{1}\left(x_{1}, y_{1}\right), \\
d(Q, R)=d(P, T)=\sqrt{a_{1}^{\prime 2} d_{1}^{2}\left(x_{1}, y_{1}\right)+a_{2}^{\prime 2} d_{2}^{2}\left(x_{2}, y_{2}\right) .}
\end{gathered}
$$

As $a_{2}^{2}-a_{2}^{\prime 2}=\lambda \beta_{2}<0$, we have $a_{2}<a_{2}^{\prime}$, so $\sqrt{a_{1}^{\prime 2} d_{1}^{2}\left(x_{1}, y_{1}\right)+a_{2}^{\prime 2} d_{2}^{2}\left(x_{2}, y_{2}\right)} \geq a_{2}^{\prime} d_{2}\left(x_{2}, y_{2}\right)>a_{2} d_{2}\left(x_{2}, y_{2}\right)$, and $\sqrt{a_{1}^{\prime 2} d_{1}^{2}\left(x_{1}, y_{1}\right)+a_{2}^{\prime 2} d_{2}^{2}\left(x_{2}, y_{2}\right)} \geq a_{1}^{\prime} d_{1}\left(x_{1}, y_{1}\right)$.

The greatest of the six "distances" is $d(Q, R)=d(P, T)=$ $\sqrt{a_{1}^{\prime 2} d_{1}^{2}\left(x_{1}, y_{1}\right)+a_{2}^{\prime 2} d_{2}^{2}\left(x_{2}, y_{2}\right)}$. In each of the four possible "triangles," $P Q R, P Q T, P R T$, and $Q R T$, there is a side equal to $\sqrt{a_{1}^{\prime 2} d_{1}^{2}\left(x_{1}, y_{1}\right)+a_{2}^{\prime 2} d_{2}^{2}\left(x_{2}, y_{2}\right)}$, another is equal to $a_{1}^{\prime} d_{1}\left(x_{1}, y_{1}\right)$, and the third is $a_{2} d_{2}\left(x_{2}, y_{2}\right)$. Therefore we have to prove only one "triangle inequality," valid for the four triangles:

$$
\begin{aligned}
& \sqrt{a_{1}^{\prime 2} d_{1}^{2}\left(x_{1}, y_{1}\right)+a_{2}^{\prime 2} d_{2}^{2}\left(x_{2}, y_{2}\right)} \\
& \quad \leq a_{1}^{\prime} d_{1}\left(x_{1}, y_{1}\right)+a_{2} d_{2}\left(x_{2}, y_{2}\right),
\end{aligned}
$$

that we square equivalently

$$
\begin{aligned}
& a_{1}^{\prime 2} d_{1}^{2}\left(x_{1}, y_{1}\right)+a_{2}^{\prime 2} d_{2}^{2}\left(x_{2}, y_{2}\right) \\
& \leq a_{1}^{\prime 2} d_{1}^{2}\left(x_{1}, y_{1}\right)+a_{2}^{2} d_{2}^{2}\left(x_{2}, y_{2}\right) \\
&+2 a_{1}^{\prime} a_{2} d_{1}\left(x_{1}, y_{1}\right) d_{2}\left(x_{2}, y_{2}\right) \\
& \Longleftrightarrow\left(a_{2}^{\prime 2}-a_{2}^{2}\right) d_{2}\left(x_{2}, y_{2}\right) \\
& \leq 2 a_{1}^{\prime} a_{2} d_{1}\left(x_{1}, y_{1}\right) \Longleftrightarrow \frac{d_{1}^{2}\left(x_{1}, y_{1}\right)}{d_{2}^{2}\left(x_{2}, y_{2}\right)} \geq \frac{\lambda^{2} \beta_{2}^{2}}{4 a_{1}^{\prime 2} a_{2}^{2}} .
\end{aligned}
$$

But from $\beta_{1} d_{1}^{2}\left(x_{1}, y_{1}\right)+\beta_{2} d_{2}^{2}\left(x_{2}, y_{2}\right)<0$ we have $d_{1}^{2}\left(x_{1}, y_{1}\right) / d_{2}^{2}\left(x_{2}, y_{2}\right)>\beta_{2} /-\beta_{1}$ and from $\lambda^{2} \beta_{1} \beta_{2}+4 a_{1}^{\prime 2} a_{2}^{2} \geq 0$ we have $\beta_{2} /-\beta_{1} \geq 4 a_{1}^{\prime 2} a_{2}^{2} / \lambda^{2} \beta_{1}^{2}$, so $d_{1}^{2}\left(x_{1}, y_{1}\right) / d_{2}^{2}\left(x_{2}, y_{2}\right) \geq$ $\lambda^{2} \beta_{2}^{2} / 4 a_{1}^{\prime 2} a_{2}^{2}$ and the triangle inequality is satisfied. So, $d$ is a distance.

We note that if $\left(E_{i}, d_{i}\right)$ are normed vector spaces, $\left(E_{i},\|\|_{i}\right)$, very slight modifications transform our main result in its normed version and that, in this case, no density condition is needed.

\section{Conflict of Interests}

The author declares that there is no conflict of interests regarding the publication of this paper.

\section{Acknowledgment}

The author would like to thank Redon A. Cabej for his valuable suggestions which improved the final version of this paper.

\section{References}

[1] D. Maldonado, "Distances, quasi-distances, doubling and nondoubling measures," Function Theory Study Seminar, Spring 2010.

[2] R. A. Macías and C. Segovia, "Lipschitz functions on spaces of homogeneous type," Advances in Mathematics, vol. 33, no. 3, pp. 257-270, 1979.

[3] B. Pepo, "Fixed points for contractive mappings of third order in pseudo-quasimetric spaces," Indagationes Mathematicae, vol. 1, no. 4, pp. 473-481, 1990.

[4] C. Peppo, "Fixed point theorems for $(\varphi, k, i, j)$-mappings," Nonlinear Analysis: Theory, Methods \& Applications, vol. 72, no. 2, pp. 562-570, 2010.

[5] C. Peppo, "Continuousquasidistances in product spaces," in Proceedings of 4th International Conference on Algebra and Functional Analysis, Elbasan, Albania, May 2010.

[6] C. Peppo, "Continuous quasidistances in product spaces2010," Albanian Journal of Natural \& Technical Science, vol. 28, 2, pp. $15-25,2010$.

[7] C. Peppo, "Distances and continuous quasi-distances in product spaces," IEEE CET, 2011. 




Advances in

Operations Research

mansans

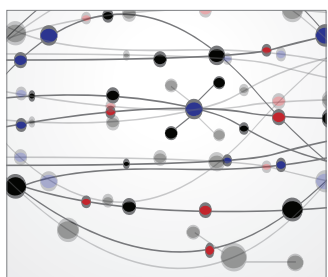

The Scientific World Journal
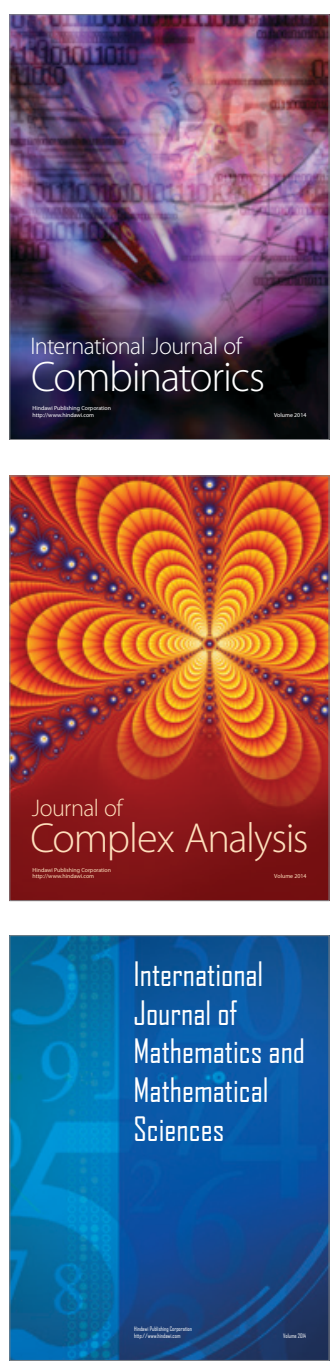
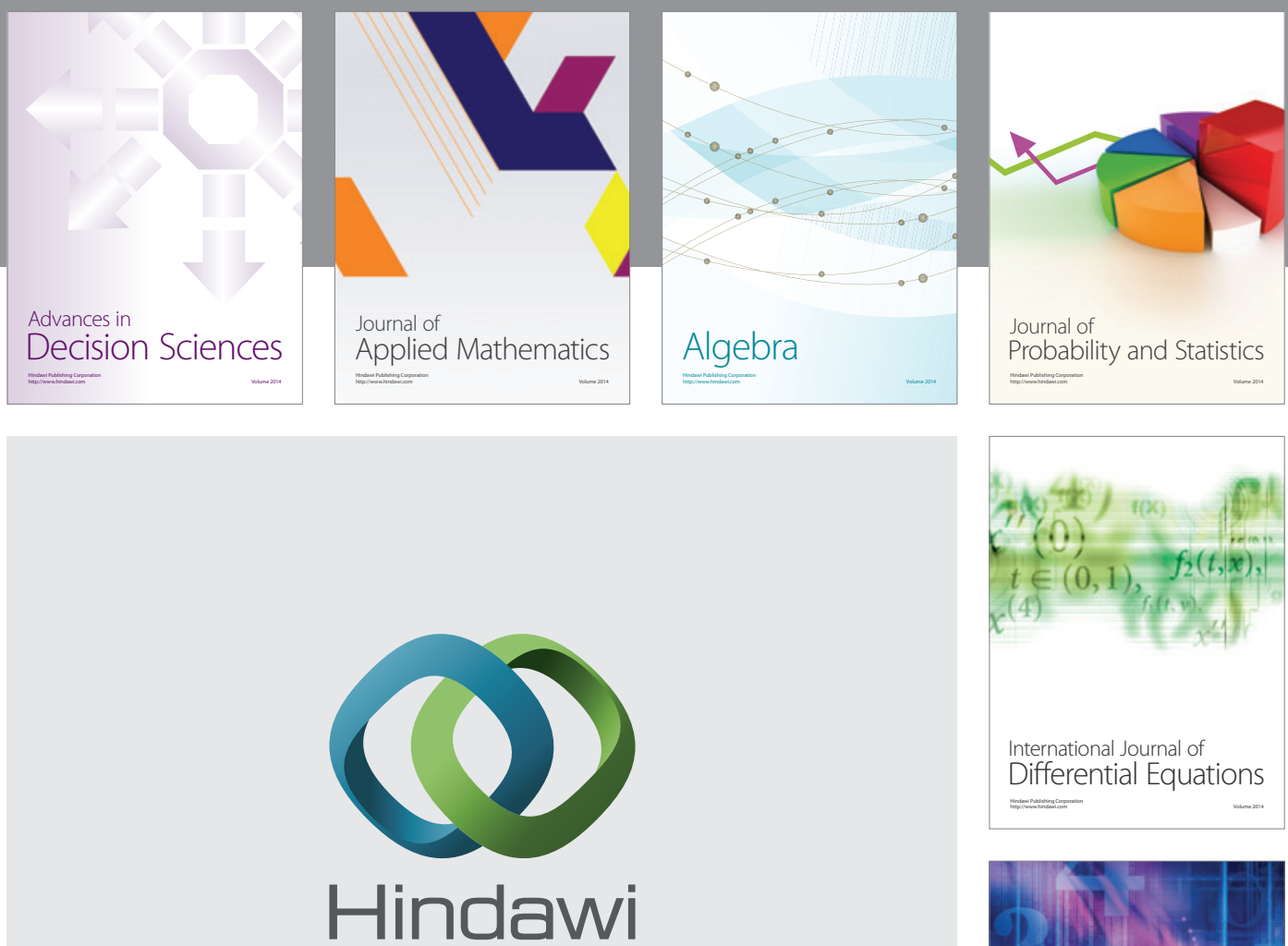

Submit your manuscripts at http://www.hindawi.com
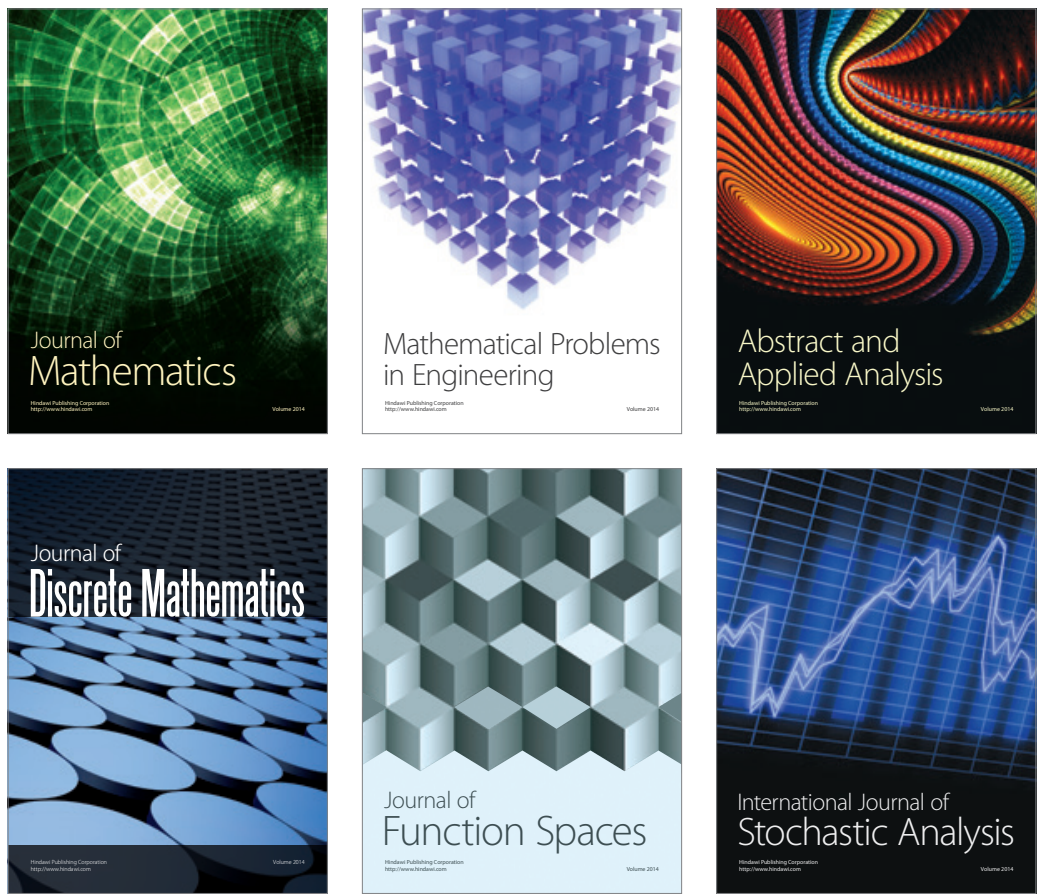

Journal of

Function Spaces

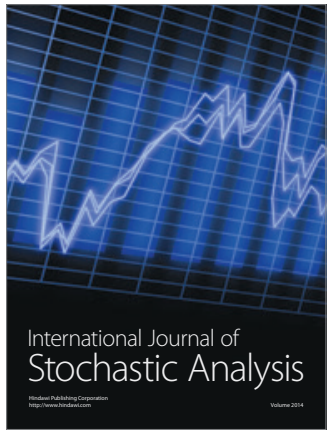

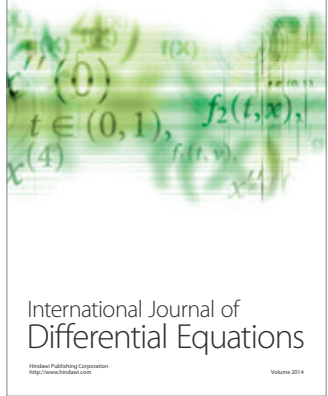
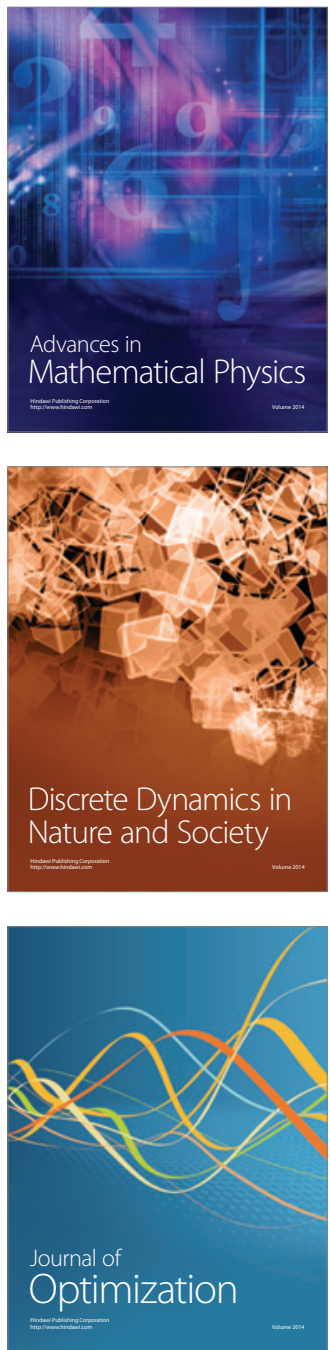